\title{
天然药物成分干预铁死亡抑制肿䈏的作用 研究进展
}

\author{
宣自学, 张轶雯, 潘宗富, 郑小卫, 黄 萍 \\ 浙江省人民医院杭州医学院附属人民医院临床药学研究中心药学部, 浙江 杭州 310014
}

[摘要] 铁死亡是一种铁依赖的程序性死亡, 不同于传统的细胞死亡方式(如调 亡、焦亡、坏死、自憡), 其特征是活性氧诱导脂质过氧化物堆积。铁死亡在肿瘤 发生发展中发挥着重要的调控作用。最新研究表明, 天然药物成分可通过谷胱甘 肽/谷胱甘肽过氧化物酶 4 途径、铁代谢、脂质代谢等调控机制诱导肿瘤细胞铁死 亡。研究发现了 30 多种天然药物成分具有诱导肿瘤细胞铁死亡的作用, 且有多通 路、多靶点的特点。本文综述了天然药物成分通过千预铁死亡抑制肿瘤的作用研 究进展。

[关键词] 铁死亡; 肿瘤; 天然药物; 中药; 调控机制; 综述

[中图分类号 ] R932 [ 文献标志码 ］ A

\section{Natural medicinal ingredients induce tumor ferroptosis and related mechanisms}

XUAN Zixue, ZHANG Yiwen, PAN Zongfu, ZHENG Xiaowei, HUANG Ping (Department of Pharmacy, Clinical Pharmacy Center, Zhejiang Provincial People's Hospital, Affiliated People's Hospital of Hangzhou Medical College, Hangzhou 310014, China)

Corresponding author: huangping@hmc.edu.cn, https://orcid.org/0000-0002-5195-7369

\begin{abstract}
[ Abstract ] Ferroptosis is an iron-dependent programmed cell death characterized by reactive oxygen species-induced lipid peroxide accumulation, which is different from cell apoptosis, pyroptosis, necrosis or autophagy. Ferroptosis plays an important role in the regulation of tumorigenesis and tumor development. Recent studies have shown that natural medicinal ingredients can induce ferroptosis in tumor cells through glutathione (GSH)/glutathione peroxidase 4 (GPx4) pathway, iron metabolism, lipid metabolism or other mechanisms. It has been reported that more than 30 natural medicinal ingredients
\end{abstract}

\section{收稿日期: 2021-07-13 接受日期:2021-09-09 网络预发表日期: 2021-10-21}

基金项目: 浙江省中医药科技计划(2018ZZ006,2021ZA006)

第一作者:宣自学,主管药师,主要从事肿瘤药理学研究;E-mail : xuanzixue0222@163.com;https://orcid.org/0000-00026296-4062

通信作者:黄 萍, 主任药师,博士生导师,主要从事肿瘤药理学研究;E-mail :huangping@hmc.edu.cn; https ://orcid.org/ 0000-0002-5195-7369 
can induce ferroptosis in tumor cells with multiple pathways and multiple targets. This article reviews the current research progress on the antitumor effects of natural medicinal ingredients through inducing cell ferroptosis.

[ Key words ] Ferroptosis; Tumor; Natural medicine; Traditional Chinese medicine; Regulatory mechanism; Review

[J Zhejiang Univ (Med Sci), 2021, 50(5): 601-606.]

[缩略语] 谷胱甘肽 (glutathione, GSH); 谷胱甘肽过氧化物酶 (glutathione peroxidase, GPx); 溶质载体家族 7 成员 11 (solute carrier family 7 member 11,SLC7A11); AMP 活化蛋白激酶(AMP-activated protein kinase, AMPK); 哺乳动物雷帕霉素靶蛋白 (mammalian target of rapamycin,mTOR); 急性髓细胞性白血病 (acute myelogenous leukemia, AML); 长链脂酰辅酶 A 合成酶 (acyl-CoA synthetase long chain family member, ACSL)

铁死亡是一种铁依赖且不同于调亡、焦亡、 自噬等传统程序性细胞死亡的细胞死亡方式, 其 特征是活性氧诱导脂质过氧化物堆积 ${ }^{[1]}$ 。铁死亡 主要表现为线粒体明显收缩、双层膜密度增加、 线粒体崉减少或消失, 但细胞膜完好、细胞核大 小正常、染色质未浓缩 ${ }^{[2]}$ 。在细胞形态和功能方 面, 铁死亡无典型坏死形态特征, 也无经典细胞 调亡特点, 且不形成自噬的封闭双层膜结构。

铁积累的增加、自由基的产生、脂肪酸供应和 脂质过氧化是诱导铁死亡的关键。目前, 铁死亡的 调控机制主要可分为三类:基于 $\mathrm{GSH} / \mathrm{GPx} 4$ 途径调 控机制、铁代谢调控机制、脂质代谢相关通路调控 机制(图 1)。研究表明, 铁死亡在肿瘤发生发展中 发挥着重要的调控作用, 已成为肿瘤机制探索及 治疗靶点研究领域的热点和焦点 ${ }^{[3]}$ 。诱导铁死亡 可能成为抗肿瘤治疗的新策略, 如 Erastin、RSL3、 索拉非尼、柳氮磺胺吡啶、铁制他汀类药物等铁死 亡诱导剂, 以及可诱导铁死亡和抑制肿瘤生长的 电离辐射和细胞因子 ${ }^{[4]}$ 。此外, 越来越多的证据 表明,多种天然药物成分可通过靶向铁死亡途径 抑制肿瘤生长。本文基于不同调控机制综述了天 然药物成分干预铁死亡发挥抗肿瘤作用的最新研 究进展, 以期为中药抗肿瘤治疗提供新思路。

\section{1 天然药物成分靶向 GSH/GPx4 途径诱导铁死亡}

半胱氨酸是合成 GSH 的限速原料, 主要由胱 氨酸还原而来, 而胱氨酸进人细胞则依赖胱氨酸/ 谷氨酸逆转运蛋白SLC7A11 ${ }^{[5]}$; 此外, GSH 是 GPx4 降解脂质过氧化物的必需反应底物。因此, 抑制
GSH/GPx4 通路可导致脂质过氧化物积累和铁死 亡发生。

目前研究发现, 新藤黄酸、猕猴桃根、青蒿琥 酯、双氢青蒿素、葫芦素 B、银杏素、二氢异丹参 酮 I、茄碱等多种天然药物成分通过干预 GSH/ GPx4途径诱导铁死亡, 进而抑制肿瘤生长。例如, 藤黄中新藤黄酸可通过p53/SLC7A $11 / G P x 4$ 信号 通路诱导转化生长因子 $\beta 1$ 刺激的黑色素瘤细胞发 生铁死亡 ${ }^{[6]}$ 。狝猴桃根可通过抑制 $\mathrm{GPx} 4$ 和 SLC7A11蛋白增加活性氧的积累, 促进胃癌细胞铁 死亡 ${ }^{[7]}$ 。三萜类化合物葫芦素 $\mathrm{B}$ 可引起人鼻咽癌 细胞内铁离子积累和 GSH 耗竭, 导致脂质过氧化 和 GPx4 表达下调, 最终引发铁死亡 ${ }^{[8]}$ 。二氢异丹 参酮 I 、茄碱也是通过抑制 $\mathrm{GPx} 4$ 分别诱导乳腺癌 细胞、肝癌细胞的铁死亡 ${ }^{[9]}$ 。银杏叶中银杏素介 导非小细胞肺癌铁死亡, 与增加铁浓度和脂质过 氧化、降低SLC7A11 和 GPx4 表达及 $\mathrm{GSH} /$ 氧化型 GSH 比值等机制相关 ${ }^{[10]}$ 。此外, 双氢青蒿素和青 蒿琥酯也可抑制 GPx4, 诱导肿瘤细胞铁死 $亡^{[11-12]}$ 。在结肠癌中, 天然产物 $\beta$-榄香烯联合西 妥昔单抗可下调铁死亡的负调控蛋白 (如 $\mathrm{GPx} 4$ 、 SLC7A11等), 促进结肠癌细胞铁死亡 ${ }^{[13]}$ 。

研究还发现,多种天然药物成分的衍生物也 能靶向 GSH/GPx4 途径, 如苯并吡喃衍生物 IMCA、 小白菊内酯的衍生物 DMOCPTL、姜黄素类似物 EF 24 和 ALZ003、喜树碱类似物 SN38 等。其中, IMCA 下调SLC7A11 的表达,降低半胱氨酸和 GSH 的含量, 导致结直肠癌活性氧积累和铁死亡 ${ }^{[14]}$; DMOCPTL通过与 GPx4 蛋白直接结合, 诱导 GPx4 


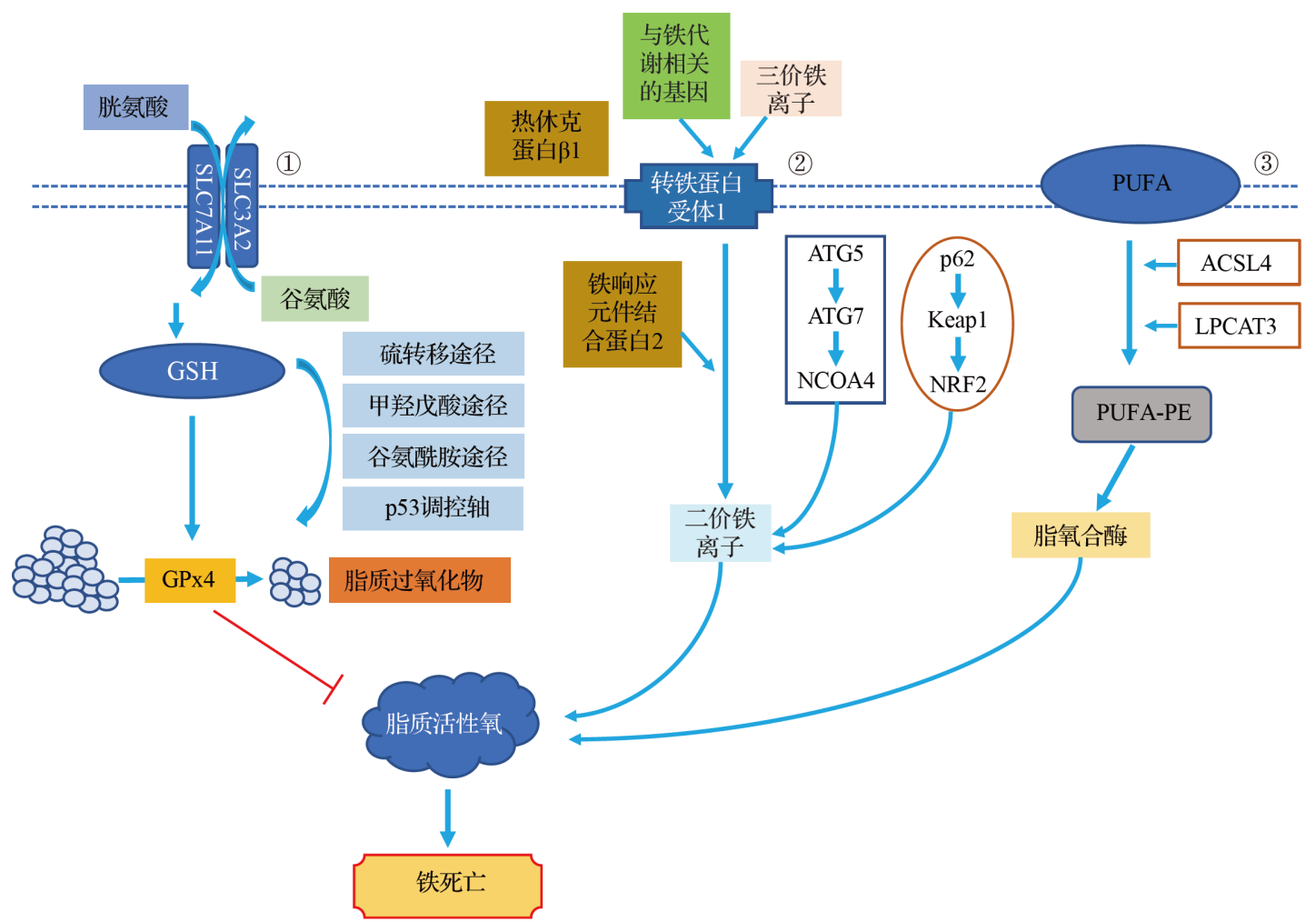

(1)SLC7A11、SLC3A2可在向细胞内转运胱氨酸的同时外排等量的谷氨酸. 朕氨酸通过还原反应可生成GSH生物合成的限速底物 半胱氨酸, GSH是GPx4的辅酶因子以及其降解脂质过氧化物的必需反应底物. GPx4作为调控铁死亡的关键酶, 可通过催化脂质过氧 化物的还原反应, 抑制铁死亡的发生. 硫转移途径、甲羟戊酸途径、谷氨酰胺途径、p53调控轴等也参与铁死亡的 GSH/GPx4途径. (2)血 液循环中的三价铁离子与转铁蛋白结合并运输, 通过细胞膜上的转铁蛋白受体 1 进人细胞内被还原为二价铁离子后, 再被转运并释 放到细胞质铁池中, 导致铁过量, 产生活性氧, 引发铁死亡. 铁蛋白代谢相关的ATG5-ATG7-NCOA4通路和p62-Keap1-NRF2通路也可 参与铁死亡过程. (3)ACSL4通过在内质网相关的氧合作用中心产生氧化的PE来促进铁死亡, 而ACSL 4 催化花生四烯酸或肾上腺素的 附着以产生酰基辅酶A衍生物, 随后通过LPCAT3将其酯化为PE, 进而被脂氧合酶氧化以产生脂质氢过氧化物, 最终导致铁死亡. SLC7A 11 : 溶质载体家族7成员 $11 ; \mathrm{SLC} 3 \mathrm{~A} 2$ : 溶质载体家族 3 成员 $2 ; \mathrm{GSH}$ : 谷胱甘肽; GPx : 谷胱甘肽过氧化物酶; ATG : 自噬相关基因; $\mathrm{NCOA}$ : 核受体辅激活因子; Keap:Kelch样环氧氯丙烷相关蛋白; NRF: 核因子E2相关因子; ACSL: 长链脂酰辅酶A合成酶; PE: 磷脂酰 乙醇胺; LPCAT : 溶血卵磷脂酰基转移酶;PUFA :多不饱和脂肪酸.

图 1 铁死亡的调控机制

Figure 1 Regulation mechanisms of ferroptosis

泛素化, 导致三阴性乳腺癌细胞铁死亡和细胞调 $亡^{[15]} ; \mathrm{EF} 24$ 显著提高丙二醛、活性氧和细胞内铁 离子水平, 抑制 $\mathrm{GPx} 4$ 表达, 诱导骨肉瘤细胞铁死 $亡^{[16]}$; ALZ003 不仅诱导胶质母细胞瘤 fbxl2 介导 的雄激素受体泛素化, 导致其降解, 还可促使细胞 中活性氧的积累、脂质过氧化和 GPx4 的抑制, 进 而诱导铁死亡 ${ }^{[17]}$; SN38 与电穿孔联合治疗可使结 肠癌细胞内超氧化物和氢过氧化物大量产生、 GSH耗竭, 诱发铁死亡 ${ }^{[18]}$ 。

\section{2 天然药物成分靶向铁代谢途径诱导铁死亡}

铁是人体中的一种重要微量元素, 二价铁离 子可转移电子给胞内氧, 使胞内氧与脂质发生反 应形成脂质过氧化物。研究发现, 双氢青蒿素能 够通过与细胞游离铁结合, 刺激铁调节蛋白与含
有铁响应元件序列的信使RNA分子结合。因此, 双氢青蒿素可破坏铁调节蛋白/铁响应元件控制 的铁稳态, 从而进一步增加细胞游离铁 ${ }^{[19]}$ 。Du 等 ${ }^{[20]}$ 发现双氢青蒿素通过调节AMPK/mTOR/ p70S6k 信号通路的活性诱导自噬, 加速铁蛋白降 解, 增加不稳定铁池, 促进细胞活性氧积累, 最终 导致 AML细胞铁死亡。过表达铁硫簇组装酶可调 节铁代谢, 降低双氢青蒿素诱导的铁死亡。另有 研究发现, 蒲花粉提取物香蒲新苗通过激活 AMPK 信号通路导致铁蛋白降解, 显著增加 AML 细胞 内和线粒体的活性氧, 进而诱导 AML 细胞铁死 $亡^{[21]}$ 。大黄素甲梄8-O- $\beta$ - 吡喃葡萄糖苷通过调控 $\mathrm{miR}-103 \mathrm{a}-3 \mathrm{p} / \mathrm{GSL} 2$ 轴, 上调活性氧、丙二醛水平以 及细胞内二价铁离子, 诱导胃癌细胞铁死亡 ${ }^{[22]}$ 。 6-姜辣素降低泛素特异性肽酶14的表达,不仅增 
加自噬体数量和活性氧水平, 而且增加铁浓度, 抑制肺癌细胞生长 ${ }^{[23]}$ 。盐霉素衍生物伊诺莫霉 素可将铁隔离在溶酶体中, 触发铁蛋白降解, 导致 铁在细胞器中进一步负荷, 进而杀死乳腺癌干 细胞 ${ }^{[24]}$ 。

综上所述, 双氢青蒿素、香蒲新苷、大黄素甲 醚8-O- $\beta$-吡喃葡萄糖苗、6-姜辣素等多种天然药物 成分可靶向铁代谢途径诱导肿瘤细胞铁死亡。

\section{3 天然药物成分靶向脂质代谢相关通路诱导铁 死亡}

脂质代谢与铁死亡密切相关。例如, 游离多 不饱和脂肪酸是铁死亡合成脂质信号转导介质的 底物, 含花生四烯酸或其衍生物肾上腺素的磷 脂酰乙醇胺是诱导细胞发生铁死亡的关键磷 脂 ${ }^{[25]}$ 。ACSL4 是调节脂质组成的关键酶, 可催化 花生四烯酸或肾上腺素的附着以产生酰基辅酶 $\mathrm{A}$ 衍生物, 溶血卵磷脂酰基转移酶 3 将其酯化为磷脂 酰乙醇胺, 进而被脂氧合酶氧化产生脂质氢过氧 化物, 最终导致铁死亡 ${ }^{[26]}$ 。

研究发现,齐墩果酸、罗波斯塔双黄酮 $\mathrm{A}$ 等天 然药物成分可靶向脂质代谢通路诱导铁死亡。齐 墩果酸是一种天然存在于植物叶片、果实和根茎 中的物质, 具有抗癌活性。齐墩果酸可增加氧化 应激水平和二价铁离子含量, 以及铁死亡相关蛋 白及 ACSL4 表达。敲低宫颈癌细胞中ACSL4 的表 达后, 齐墩果酸抗癌作用被抵消, 活性氧和 GPx4 水平下降, 提示齐墩果酸通过促进 ACSL4 的表达 来激活宫颈癌细胞的铁死亡 ${ }^{[27]}$ 。另一项研究从 毛卷柏中分离得到 6种 C-3'-C-6 双黄酮, 其中罗波 斯塔双黄酮 $\mathrm{A}$ 抗乳腺癌的活性最强, 其机制为通 过增强电压依赖性阴离子通道 2 表达, 降低 Nedd4 E3 泛素连接酶的表达, 导致脂质过氧化和活性氧 的产生, 从而诱发乳腺癌细胞铁死亡 ${ }^{[28]}$ 。此外, 椞木科Betula etnensis Raf的树皮甲醇提取物可增 加结肠癌细胞脂质过氧化和血红素加氧酶 1 、活性 氧表达, 促进细胞铁死亡 ${ }^{[29]}$ 。

\section{4 天然药物成分通过其他途径诱导铁死亡}

天然药物成分不仅可通过 $\mathrm{GSH} / \mathrm{GPx} 4$ 、铁代 谢、脂质代谢调控机制诱导肿瘤细胞铁死亡, 还可 能通过其他途径调控铁死亡。例如, Du 等 ${ }^{[30]}$ 研究 发现舒肝宁注射液通过血红素加氧酶 1 诱导三阴
性乳腺癌细胞铁死亡。青蒿琥酯激活 A TF 4CHOP-CHAC1 通路导致伯基特淋巴瘤细胞铁死 $亡^{[31]}$ 。最近有研究发现, 天然药物成分可抑制多 药耐药肿瘤细胞生长也与铁死亡相关。如藤黄属 植物苯甲酮类化合物 Epunctanone 通过改变基质 金属蛋白酶和增加活性氧的产生诱导多药耐药肿 瘤细胞的铁死亡 ${ }^{[32]}$; 天然齐墩烷型三萜皇苷 Ardisiacrispin B 能够通过铁死亡和细胞凋亡抑制 多药耐药肿瘤细胞的生长 ${ }^{[33]}$, 但具体机制有待进 一步探索。

此外, 还有一些天然药物可促进肿瘤细胞铁 死亡, 但机制也不明确。例如, 槐耳水提液诱导非 小细胞肺癌细胞铁死亡 ${ }^{[34]}$; 穿心莲的抗结直肠癌 和化疗增敏作用部分依赖铁死亡的激活 ${ }^{[35]}$; 百里 香和牛蒡提取物可通过诱导细胞铁死亡抑制白血 病和多发性骨髓瘤的细胞增殖 ${ }^{[36]}$; 药用球果紫堇 的氯仿提取物可诱导多发性骨髓瘤细胞铁死 $亡^{[37]}$; 苦瓜种子中分离的生物活性蛋白 MAP30在 体内通过改变代谢和诱导铁死亡, 与顺铂协同发 挥抑制卵巢癌的作用 ${ }^{[38]}$ 。

\section{5 结 语}

目前已发现多种天然药物成分可诱导肿瘤细 胞铁死亡, 且具有多通路、多靶点的特点。然而, 当前的研究多数停留在细胞水平, 缺乏动物及临 床水平的验证, 因此天然药物成分干预铁死亡的 作用有待进一步探索。此外,随着研究的不断推 进,相信天然药物成分诱导肿瘤细胞铁死亡的作 用机制将越来越丰富。

利益冲突 所有作者均声明不存在利益冲突

\section{参考文献}

[1] DIXON S J, LEMBERG K M, LAMPRECHT M R, et al. Ferroptosis: an iron-dependent form of nonapoptotic cell death [J]. Cell, 2012, 149(5): 1060-1072.

[2] STOCKWELL B R, FRIEDMANN ANGELI J P, BAYIR $\mathrm{H}$, et al. Ferroptosis: a regulated cell death nexus linking metabolism, redox biology, and disease $[\mathrm{J}]$. Cell, 2017, 171(2): 273-285.

[3] MOU Y, WANG J, WU J, et al. Ferroptosis, a new form of cell death: opportunities and challenges in cancer $[\mathrm{J}]$. J Hematol Oncol, 2019, 12(1): 34.

[4] XIA X, FAN X, ZHAO M, et al. The relationship between ferroptosis and tumors: a novel landscape for therapeutic approach $[J]$. Curr Gene Ther, 2019, 
19(2): 117-124.

[5] URSINI F, MAIORINO M. Lipid peroxidation and ferroptosis: the role of GSH and GPx4[J]. Free Radic Biol Med, 2020, 152: 175-185.

[6] WANG M, LI S, WANG Y, et al. Gambogenic acid induces ferroptosis in melanoma cells undergoing epithelial-to-mesenchymal transition $[\mathrm{J}]$. Toxicol Appl Pharmacol, 2020, 401: 115110.

[7] GAO Z, DENG G, LI Y, et al. Actinidia chinensis planch prevents proliferation and migration of gastric cancer associated with apoptosis, ferroptosis activation and mesenchymal phenotype suppression $[\mathrm{J}]$. Biomed Pharmacother, 2020, 126: 110092.

[8] HUANG S, CAO B, ZHANG J, et al. Induction of ferroptosis in human nasopharyngeal cancer cells by cucurbitacin B: molecular mechanism and therapeutic potential $[J]$. Cell Death Dis, 2021, 12(3): 237.

[9] LIN Y S, SHEN Y C, WU C Y, et al. Danshen improves survival of patients with breast cancer and dihydroisotanshinone I induces ferroptosis and apoptosis of breast cancer cells $[\mathrm{J}]$. Front Pharmacol, 2019, 10: 1226.

[10] LOU J S, ZHAO L P, HUANG Z H, et al. Ginkgetin derived from Ginkgo biloba leaves enhances the therapeutic effect of cisplatin via ferroptosis-mediated disruption of the Nrf2/HO-1 axis in EGFR wild-type non-small-cell lung cancer $[\mathrm{J}]$. Phytomedicine, 2021, 80: 153370 .

[11] ROH J L, KIM E H, JANG H, et al. Nrf2 inhibition reverses the resistance of cisplatin-resistant head and neck cancer cells to artesunate-induced ferroptosis $[\mathrm{J}]$. Redox Biol, 2017, 11: 254-262.

[12] CHEN G Q, BENTHANI F A, WU J, et al. Artemisinin compounds sensitize cancer cells to ferroptosis by regulating iron homeostasis $[\mathrm{J}]$. Cell Death Differ, 2020, 27(1): 242-254.

[13] CHEN P, LI X, ZHANG R, et al. Combinative treatment of $\beta$-elemene and cetuximab is sensitive to KRAS mutant colorectal cancer cells by inducing ferroptosis and inhibiting epithelial-mesenchymal transformation $[\mathrm{J}]$. Theranostics, 2020, 10(11): 5107-5119.

[14] ZHANG L, LIU W, LIU F, et al. IMCA induces ferroptosis mediated by SLC7A11 through the AMPK/ mTOR pathway in colorectal cancer $[\mathrm{J}]$. Oxid Med Cell Longev, 2020, 2020: 6901472.

[15] DING Y, CHEN X, LIU C, et al. Identification of a small molecule as inducer of ferroptosis and apoptosis through ubiquitination of GPX4 in triple negative breast cancer cells $[\mathrm{J}]$. J Hematol Oncol, 2021, 14(1): 19.

[16] LIN H, CHEN X, ZHANG C, et al. EF24 induces ferroptosis in osteosarcoma cells through HMOX1[J]. Biomed Pharmacother, 2021, 136: 111202.

[17] CHEN T C, CHUANG J Y, KO C Y, et al. AR ubiquitination induced by the curcumin analog suppresses growth of temozolomide-resistant glioblastoma through disrupting GPX4-mediated redox homeostasis[J]. Redox Biol, 2020, 30: 101413.

[18] NIKOLOVA B, SEMKOVA S, TSONEVA I, et al. Redox-related molecular mechanism of sensitizing colon cancer cells to camptothecin analog $\mathrm{SN} 38[\mathrm{~J}]$. Anticancer Res, 2020, 40(9): 5159-5170.

[19] OOKO E, SAEED M E M, KADIOGLU O, et al. Artemisinin derivatives induce iron-dependent cell death (ferroptosis) in tumor cells $[\mathrm{J}]$. Phytomedicine, 2015, 22(11): 1045-1054.

[20 ] DU J, WANG T, LI Y, et al. DHA inhibits proliferation and induces ferroptosis of leukemia cells through autophagy dependent degradation of ferritin $[\mathrm{J}]$. Free Radical Biol Med, 2019, 131: 356-369.

[21] ZHU H Y, HUANG Z X, CHEN G Q, et al. Typhaneoside prevents acute myeloid leukemia (AML) through suppressing proliferation and inducing ferroptosis associated with autophagy $[\mathrm{J}]$. Biochem Biophysl Res Commun, 2019, 516(4): 1265-1271.

[22] NIU Y, ZHANG J, TONG Y, et al. Physcion 8-O- $\beta$ glucopyranoside induced ferroptosis via regulating miR-103a-3p/GLS2 axis in gastric cancer[J]. Life Sci, 2019, 237: 116893.

[23] TSAI Y, XIA C, SUN Z. The inhibitory effect of 6gingerol on ubiquitin-specific peptidase 14 enhances autophagy-dependent ferroptosis and anti-tumor in vivo and in vitro $[\mathrm{J}]$. Front Pharmacol, 2020, 11: 598555.

[24] MAI T T, HAMAÏ A, HIENZSCH A, et al. Salinomycin kills cancer stem cells by sequestering iron in lysosomes[J]. Nat Chem, 2017, 9(10): 1025-1033.

[25] KAGAN V E, MAO G, QU F, et al. Oxidized arachidonic and adrenic PEs navigate cells to ferroptosis[J]. Nat Chem Biol, 2017, 13(1): 81-90.

[26] DIXON S J, WINTER G E, MUSAVI L S, et al. Human haploid cell genetics reveals roles for lipid metabolism genes in nonapoptotic cell death $[\mathrm{J}]$. ACS Chem Biol, 2015, 10(7): 1604-1609.

[27] XIAOFEI J, MINGQING S, MIAO S, et al. Oleanolic acid inhibits cervical cancer HeLa cell proliferation through modulation of the ACSL4 ferroptosis signaling pathway $[\mathrm{J}]$. Biochem Biophysl Res Commun, 2021, 545: 81-88.

[28] XIE Y, ZHOU X, LI J, et al. Identification of a new natural biflavonoids against breast cancer cells induced ferroptosis via the mitochondrial pathway $[\mathrm{J}]$. Bioorg Chem, 2021, 109: 104744.

[29] MALFA G A, TOMASELlO B, ACQUAVIVA R, et al. Betula etnensis Raf. (betulaceae) extract induced HO-1 expression and ferroptosis cell death in human colon cancer cells[J]. Int J Mol Sci, 2019, 20(11): 2723.

[30] DU J, WANG L, HUANG X, et al. Shuganning 
injection, a traditional Chinese patent medicine, induces ferroptosis and suppresses tumor growth in triple-negative breast cancer cells $[\mathrm{J}]$. Phytomedicine, 2021, 85: 153551.

[31] WANG N, ZENG G Z, YIN J L, et al. Artesunate activates the ATF4-CHOP-CHAC1 pathway and affects ferroptosis in burkitt's lymphoma $[\mathrm{J}]$. Biochem Biophysl Res Commun, 2019, 519(3): 533-539.

[32] MBAVENG A T, FOTSO G W, NGNINTEDO D, et al. Cytotoxicity of epunctanone and four other phytochemicals isolated from the medicinal plants garcinia epunctata and ptycholobium contortum towards multi-factorial drug resistant cancer cells $[\mathrm{J}]$. Phytomedicine, 2018, 48: 112-119.

[33] MBAVENG A T, NDONTSA B L, KUETE V, et al. A naturally occuring triterpene saponin ardisiacrispin $\mathrm{B}$ displayed cytotoxic effects in multi-factorial drug resistant cancer cells via ferroptotic and apoptotic cell death $[\mathrm{J}]$. Phytomedicine, 2018, 43: 78-85.

[34] 田颖颖,杨爱琳, 陈孝男, 等. 槐耳清膏抑制人非小 细胞肺癌 NCI-H1299 细胞生长和转移及其作用机 制研究 $[J]$. 中国中药杂志, 2020, 45(15): 37003706.

TIAN Yingying, YANG Ailin, CHEN Xiaonan, et al. Effect of Huaier aqueous extract on growth and metastasis of human non-small cell lung cancer NCI-
H1299 cells and its underlying mechanisms $[\mathrm{J}]$. China Journal of Chinese Materia Medica, 2020, 45(15): 3700-3706. (in Chinese)

[35] SHARMA P, SHIMURA T, BANWAIT J K, et al. Andrographis-mediated chemosensitization through activation of ferroptosis and suppression of $\beta$-catenin/ Wnt-signaling pathways in colorectal cancer $[\mathrm{J}]$. Carcinogenesis, 2020, 41(10): 1385-1394.

[36] ADHAM A N, HEGAZY M E F, NAQISHBANDI A M, et al. Induction of apoptosis, autophagy and ferroptosis by thymus vulgaris and arctium lappa extract in leukemia and multiple myeloma cell lines $[\mathrm{J}]$. Molecules, 2020, 25(21): 5016.

[37] ADHAM A N, NAQISHBANDI A M, EFFERTH T. Cytotoxicity and apoptosis induction by fumaria officinalis extracts in leukemia and multiple myeloma cell lines $[J]$. J Ethnopharmacol, 2021, 266: 113458.

[38] CHAN D W, YUNG M M, CHAN Y S, et al. MAP30 protein from Momordica charantia is therapeutic and has synergic activity with cisplatin against ovarian cancer in vivo by altering metabolism and inducing ferroptosis [J]. Pharmacol Res, 2020, 161: 105157.

$$
\text { [本文编辑 沈 敏 余 方] }
$$

\section{陈静海研究员团队揭示长链非编码RNA在心脏代谢 及心脏重构中的作用}

近日,浙江大学转化医学研究院和浙江大学医学院附属第二医院陈静海研究员联合哈佛医学院波士顿儿童医院王 大志教授等在 《心脏病学基础研究》 (Basic Research in Cardiology) 发表题为 “LncRNA LncHrt preserves cardiac metabolic homeostasis and heart function by modulating the LKB1-AMPK signaling pathway” 的研究论文(https://doi. org/10. 1007/ s00395-021-00887-3), 报道了心肌细胞富集的长链非编码RNA LncHrt通过调节SIRT2-LKB1-AMPK 激酶信号级联反应, 保护心肌梗死后心脏能量代谢稳态, 从而改善心脏缺血损伤后的结构和功能。

研究人员发现,心肌梗死后心脏中长链非编码RNA LncHrt表达显著下调,CDK5与SIRT2的相互作用增加,并伴随着 LKB1-AMPK信号通路的抑制。在心肌梗死心脏中通过AAV9补偿LncHrt的表达,LncHrt通过干扰CDK5对SIRT2活性的抑 制，促进SIRT2下游的LKB1-AMPK信号通路的激活。激活的AMPK调控心脏的能量代谢稳态，进而缓解心肌梗死后心脏 的病理学重构,保护心脏功能,阻止心力衰竭的发生。该研究鉴定了心肌富集的 LncHrt 为心脏代谢的调节分子,其通过 调控代谢信号通路, 保护心脏损伤后的结构与功能。并且AAV9介导的LncHrt可以作为基于RNA治疗缺血性心脏疾病的 有效靶点。

刘宁博士研究生、Masaharu Kataoka博士和王迎超博士研究生为论文共同第一作者。研究工作得到国家重点研发计 划、国家自然科学基金、浙江省自然科学基金和浙江大学转化医学研究院种子基金等资助。 